\title{
A facilitating effect of latent extinction in a partial reinforcement situation
}

\author{
ELVIS C. JONES, RICHARD L. NARVER AND CECIL C. BRIOGES, JR. \\ TEXAS CHRISTIAN UNIVERSITY
}

In two experiments, rats were trained on a fixed-ratio partial reinforcement schedule in a runway. Following training, the $S$ were given one latent extinction plucement in the goal box prior to the ir regular running trials. In the first experiment, 5 min. intervened between the latent extinction placement and the first running trial, and testing occurred on two days. In the second experiment, Ss were started in the runway immediately following the latent extinction placement, and all test trials were run on the same day. In both experiments, the latent extinction placement decreased running speeds initially, but on subsequent trials performance was enhanced. It was concluded that temporary facilitation may be expected to occur when mild latent extinction is given following training on fixed-ratio schedules.

Latent extinction is known to produce greater decrements in responding than does regular response extinction. Dyal (1962) has found that within 3-4 trials this decrement tends to be overcome, such that latent extinction Ss do not significantly differ from Ss which did not receive latent extinction placements. The Ss in Dyal's experiment were trained on a consistent reinforcement schedule. Dyal (1965) has also found that prior training on a partial reinforcement schedule (variable-ratio) attenuates the effects of latent extinction.

It may be that the effects of latent extinction placements are not essentially different from a regular response extinction trial, except possibly more effective for a short period of time. The present experiments evaluated this hypothesis.

In a straight runway, when Ss have been trained on a fixed-ratio schedule, they tend to mun faster on later trials than on the first trial. If a latent extinction placement is not unlike a regular nonreinforced trial, then following a latent extinction placement, Ss should run faster on the first regular trial than they would had they not received the latent extinction placement.

\section{Experiment 1.}

\section{METHOD}

The Ss were 30 female Sprague-Dawley rats, 100 days old at the start of the experiment. The Ss were trained in a straight runway which was 48 in. long, 4-1/2 in. wide, and 6 in. high, excluding the goal box. The goal box was 10 in. $x$ 4-1/2 in. $x 6$ in., and contained a copper food cup, 2 in. in diameter and $1 / 2$ in. deep. The runway floor was a bar grid.

All Ss were maintained on a $23-\mathrm{hr}$. food deprivation schedule and given three training trials in the runway each day for 10 days. A 5-min. intertrial interval was used. Only the third trial on each day was reinforced with five Noyes Precision Food Pellets. On all trials the Ss were confined in the goal box for $30 \mathrm{sec}$., then removed to their home cages to await the next trial. Water, but no food, was present in the home cages at all times. Fifteen min. after the last trial of each day, the Ss were fed for one hour. On the basis of mean latencies on the three trials of day 10, the Ss were assigned to two groups in a randomized blocks design.

On the eleventh day, Ss in the $\mathrm{E}$ group were placed in the empty goal box for $30 \mathrm{sec}, 5 \mathrm{~min}$. prior to running their regular trials. Ss in the $\mathrm{C}$ group ran their three regular trials without a latent extinction placement. The following day, all Ss were given three additional trials without further latent extinction placements. Reinforcement was given on the third trial on both days.

\section{Experiment II.}

Experiment II differed from Experiment I only in the following ways: There were six Ss in each group. After 11 days of training, Ss in the $E$ group were placed in the empty goal box for $30 \mathrm{sec}$., then were immediately placed at the start of the runway and given their first regular trial. Ss in both groups received five trials on the same day and reinforcement was omitted entirely.

\section{RESULTS AND DISCUSSION}

As indicated by Fig. 1, E Ss in Experiment I did tend to run faster than did the $\mathrm{C} S \mathrm{Ss}(\mathrm{F}=4.30, \mathrm{df}=1 / 28$,

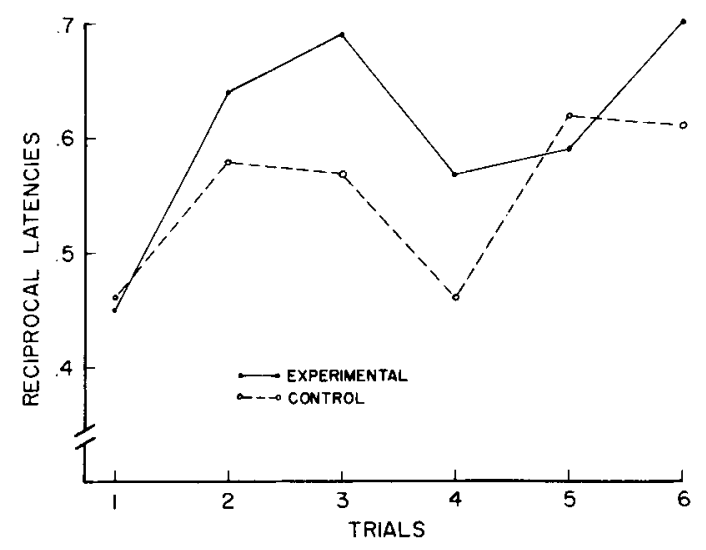

Fig. 1. Runway performance in Experiment 1. One group received a latent extinction placement (experimental group) prior to the first runway trial. The control group received no latent extinction placements. The first 3 and the last 3 trials occurred on successive days. 


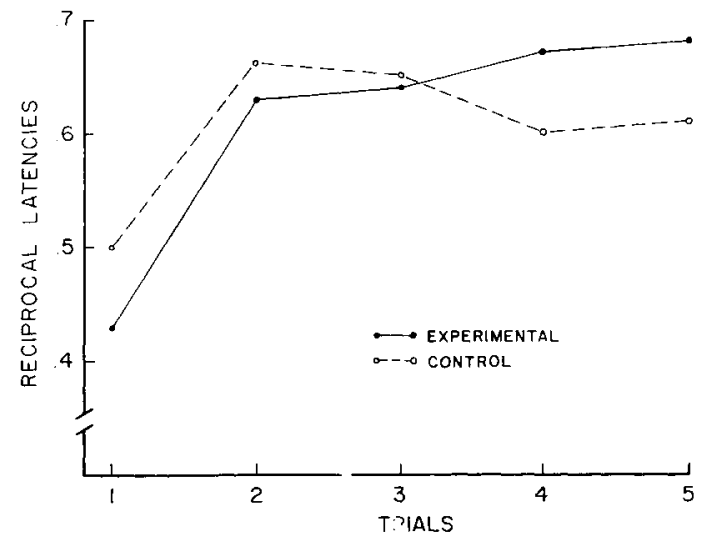

Fig. 2. Runway performance in Experiment 11 . The experimental group received a latent extinction placement prior to the first runway trial. The control group received no latent extinction placements. All trials were given on the same day.

$\mathrm{p}<.05$ ), but not on the first trial, contrary to the hypothesis. Trials 1-3 and 4-6 represented different days, so the facilitating effect of the latent extinction placement carried over from one day to the next. Trend analysis over all six trials indicated the groups differed in trend $(F=2.35, \mathrm{df}=5 / 140, p<.05)$.

Figure 2 illustrates that with a shorter interval between the latent extinction placement and the first regular trial, the initial decrement in responding was stronger. It wasn't until the fourth trial that the $\mathbf{E}$ group ran faster than the $\mathrm{C}$ group. The $\mathrm{E}$ group did, however, eventually run faster than did the C group, indicating that facilitation was only retarded by the more severe latent extinction procedure. The groups differed in trend over the five test trials $(F=2.49$, $\mathrm{df}=4 / 44, \mathrm{p}<.05$ ).

The slow running on trials 1 and 4 in both experiments is no doubt a function of the fixed-ratio schedule upon which the Ss had been trained. In Experiment II, the eventual superiority of the experimental group was partly in the form of resistance to suppression. One result of latent extinction placements may, therefore, be to break up the patterning of responding, making Ss conform more to variable-ratio responding, rather than fixed-ratio responding.
The data suggest that a latent extinction placement is not equivalent to a regular unreinforced trial; otherwise facilitation would have occurred on the first trial and there would be no way to account for differences beyond the third trial.

Most (if not all) prior research has differed from the present experiments in at least one of the following ways: (1) several latent extinction placements were given, (2) placements were of long durations (especially relative to duration of confinements during acquisition), or (3) short periods intervened between the placements and the regular running trials. Thus, information is lacking on the effects of mild latent extinction procedures, especially as they relate to the specific reinforcement schedule used in the present experiments. However, Dyal (1962) found that following an initial decrement in responding after latent extinction placement, all of his groups ran faster on subsequent extinction trials, eventually "catching up" with the control groups. His Ss were trained under consistent reinforcement and received from 1 to 10 placements of 30 to $60 \mathrm{sec}$. duration. There was a 60 to $90 \mathrm{sec}$. period between placement(s) and the first running trial. During acquisition, the Ss remained in the goal box for only 10 to $15 \mathrm{sec}$.

In a frustration theory context, it is possible (at least for partial reinforcement schedules) that since frustration has already become attached to running in the process of acquisition, mild latent extinction will not only have shorter decremental effects, but will eventually facilitate responding as in the present experiments. By "mild" latent extinction, reference is to the number of placements, the duration of placements (especially relative to goal box confinements during acquisition), intervals between placements, and the interval between the last placement and the first running trial. These variables are not seen as necessarily additive in their effects, and not necessarily linearly related to performance.

\section{References}

Dyal, J. A. Latent extinction as a function of number and duration of pre-extinction exposures. J. exp. Psychol., 1962, 63, 98-104.

Dyal, J. A. Latent extinction as a function of partial reinforcement of the running response. Psychon. Sci., 1965, 3, 207-208. 